\title{
BMJ Open Pharmacological interventions for the prevention of fetal growth restriction: protocol for a systematic review and network meta-analysis
}

\author{
Alessandra Bettiol, ${ }^{1}$ Niccolò Lombardi, ${ }^{1}$ Giada Crescioli, ${ }^{1}$ Laura Avagliano, ${ }^{2}$ \\ Alessandro Mugelli, ${ }^{1}$ Claudia Ravaldi, ${ }^{3,4}$ Alfredo Vannacci ${ }^{\odot 1,4}$
}

To cite: Bettiol A,

Lombardi N, Crescioli G, et al. Pharmacological interventions for the prevention of fetal growth restriction: protocol for a systematic review and network meta-analysis. BMJ Open 2019;9:e029467. doi:10.1136/ bmjopen-2019-029467

\section{- Prepublication history and} additional material for this paper are available online. To view these files, please visit the journal online (http://dx.doi. org/10.1136/bmjopen-2019029467).

Received 28 January 2019 Revised 15 May 2019 Accepted 21 May 2019

Check for updates

(C) Author(s) (or their employer(s)) 2019. Re-use permitted under CC BY-NC. No commercial re-use. See rights and permissions. Published by BMJ.

${ }^{1}$ Department of Neurosciences, Psychology, Drug Research and Child Health, University of Florence, Florence, Italy ${ }^{2}$ Department of Health Sciences, San Paolo Hospital Medical School, Università degli Studi di Milano, Milano, Italy

${ }^{3}$ Department of Health Sciences, University of Florence, Florence, Italy

${ }^{4}$ CiaoLapo - Charity for Healthy Pregnancy, Stillbirth and Perinatal Loss Support, Prato, Italy

Correspondence to

Dr Alfredo Vannacci;

alfredo.vannacci@unifi.it

\section{ABSTRACT}

Introduction Fetal growth restriction (FGR) includes different conditions in which a fetus fails to reach the own full growth, and accounts for $28 \%-45 \%$ of non-anomalous stillbirths. The management of FGR is based on the prolongation of pregnancy long enough for fetal organs to mature while preventing starvation. As for pharmacological management, most guidelines recommend treatment with low-dose aspirin and/or with heparin, although this approach is still controversial and innovative promising therapies are under investigation. As no firm evidence exists to guide clinicians towards the most effective therapeutic intervention, this protocol describes methods for a systematic review and network meta-analysis (NetMA) of pharmacological treatments for FGR prevention.

Methods and analysis We will search MEDLINE and Embase for clinical trials and observational studies performed on gestating women with clinically diagnosed risk of FGR. Experimental interventions will include heparin and low-molecular-weight heparin, acetylsalicylic acid, antiplatelet agents, phosphodiesterase type 3 and 5 inhibitors, maternal vascular endothelial growth factor gene therapy, nanoparticles, microRNA, statins, nitric oxide donors, hydrogen sulphide, proton pump inhibitors, melatonin, creatine and $\mathrm{N}$-acetylcysteine, and insulinlike growth factors, compared between each other or to placebo or no treatment. Primary efficacy outcome is FGR. Secondary efficacy outcomes will be preterm birth, fetal or neonatal death and neonatal complications. For the safety outcome, all adverse events reported in included studies and experienced by either mothers, fetuses or newborns will be considered. Two review authors will independently screen title, abstract and full paper text, and will independently extract data from included studies. Where possible and appropriate, for primary and secondary efficacy outcomes, a NetMA will be performed using a random-effects model within a frequentist framework. Adverse events will be narratively described.

Ethics and dissemination Results will be disseminated through a peer-reviewed scientific journal, and by scientific congresses and meetings.

PROSPERO registration number CRD42019122831.
Strengths and limitations of this study

Both clinical trials and observational studies will be included.

- Where possible, results will be synthetised using a network meta-analysis, thus allowing to simultaneously combine both direct and indirect evidence.

- The study team includes clinicians, pharmacologists and experts in the field of pregnancy complications and related therapeutic interventions.

\section{INTRODUCTION}

Fetal growth restriction (FGR), also known as intrauterine growth restriction, includes different conditions in which a fetus fails to reach its own growth potential. For many years, the most common parameter used to measure FGR was small for gestational age (SGA), ${ }^{1}$ although the two terms are not synonymous. Recently, an international clinical consensus was obtained about the definition of $\mathrm{FGR}^{2}$; therefore, works aimed to study the risk of growth-related adverse fetal outcome should focus on true FGR. Namely, SGA is usually defined by a fetal size $<10$ th centile for a population or customised standard. ${ }^{1}$ However, this definition includes also a proportion of constitutionally small but health fetuses while excluding a group of fetuses with biometry $>10$ th percentile but not meeting their own growth potential. On the other hand, the concept of FGR applies only to non-healthy fetuses that failed to reach their growth potential and thus are at increased risk of adverse outcomes. ${ }^{2}$

According to the definition identified through a Delphi consensus procedure, ${ }^{2}$ early FGR (ie, at gestational age $<32$ weeks), in the absence of congenital anomalies, is defined by fetal abdominal circumference (AC)/ estimated fetal weight (EFW) $<3$ rd centile or umbilical artery (UA) absent end-diastolic 
flow, or by the co-presence of AC $(\mathrm{EFW}<10$ th centile and uterine artery pulsatility index (PI) $>95$ th centile, alone or in combination with UA-PI $>95$ th centile. On the other hand, late FGR (ie, at gestational age of 32 weeks of more), is defined, in the absence of congenital anomalies, by $\mathrm{AC} / \mathrm{EFW}<3$ rd centile or by the co-presence of at least two parameters among (i) AC/EFW $<10$ th centile, (ii) $\mathrm{AC} / \mathrm{EFW}$ crossing centiles $>2$ quartiles on growth centiles or (iii) cerebroplacental ratio $<5$ th centile or UA-PI $>95$ th centile.

Suboptimal fetal growth accounts for $28 \%-45 \%$ of cases of non-anomalous stillbirth. ${ }^{34}$

Causes of FGR can be fetus-related or mother-related: among the former, there are chromosomal anomalies, genetic syndromes and infections. Among maternal conditions, the most common are clinically relevant conditions such as autoimmune disorders, clinically relevant conditions such as autoimmune disorders, hypoxemic conditions (such as severe anaemia, congenital cyanotic heart diseases), cardiovascular diseases (such as hypertension) or the exposition to environmental toxins. The mechanism leading to FGR involves an abnormal trophoblast invasion of the maternal spiral arteries during pregnancy, which results in an incomplete remodelling of these vessels and in the persistence of a high-resistance and low-flow uteroplacental circulation, which on its turn determines and insufficient gaseous and nutrient exchange for optimal fetal growth. ${ }^{5}$

This results in a cascade of events, including reduced placental perfusion, imbalance in angiogenic factors, and in vascular endothelial growth factor (VEGF) and placental growth factor, and may lead to placenta-mediated complications of pregnancy such as FGR, preeclampsia, placental abruption and late pregnancy loss. ${ }^{67}$

The management of FGR is based on the prolongation of pregnancy long enough for fetal organs to mature while avoiding irreversible fetus' sufferance. ${ }^{8}$ As for pharmacological management, most guidelines recommend treatment with low-dose aspirin-in preference by gestational week 16-for prevention of FGR, ${ }^{9-12}$ although this approach is not universally accepted. ${ }^{13}$ The use of heparin is also controversial: the Canadian guideline recommends that heparin should be offered in selected women, ${ }^{9}$ although recent evidence indicates that enoxaparin is not effective in preventing FGR in women with previous severe or early-onset FGR, or in those with thrombophilia, and can therefore not be recommended for this purpose. ${ }^{114}$ Furthermore, several other promising therapies are currently under investigation. ${ }^{1}$

To date, given that no firm evidence exists to guide clinicians towards the most effective therapeutic intervention, planned early birth is recommended and offered once a fetus reaches a viable gestational age and size. However, preterm birth is further associated with a consistent risk of morbidity and mortality. ${ }^{1}$

In light of the above-mentioned lack in knowledge, we aim to perform a systematic review and network meta-analysis (NetMA) of clinical trials and observational studies conducted on gestating women at diagnosed risk of FGR, with the primary objective of estimating the effect of different pharmacological treatments on the incidence of FGR.

Secondary efficacy objectives include the evaluation of the effect of different therapeutic strategies on the incidence of (i) preterm birth; (ii) fetal or neonatal death and (iii) neonatal complications related to the abnormal growth. Safety objective includes the evaluation of adverse events involving treated women, fetuses or newborns.

\section{METHODS AND ANALYSIS}

This protocol has been written according to the Preferred Reporting Items for Systematic Review and Meta-Analysis Protocols guidance. ${ }^{15}$

\section{Eligibility criteria}

Studies will be selected according to the eligibility criteria outlined below.

\section{Study designs}

We will consider for inclusion both clinical trials and observational cohort studies, either prospective or retrospective. Observational cross-sectional studies or casecontrol studies will be excluded. Similarly, we will exclude reviews and meta-analyses, letters to the editor, case reports, case series and expert opinions.

\section{Participants}

We will include studies performed on gestating women at diagnosed risk of FGR, including ${ }^{16}$ the following:

- History of late pregnancy loss or FGR.

- History of recurrent pregnancy loss (define by three or more consecutive first trimester spontaneous abortions).

- Hypertensive diseases.

- Pre-eclampsia (in the current or previous pregnancies).

- Diabetes mellitus.

- Congenital cyanotic heart diseases.

- Restrictive pneumopathies.

- Severe renal diseases.

- Autoimmune diseases.

- Hereditary or acquired thrombophilia.

- Severe anaemia.

Other risk factors for FGR reported by the clinicians as present in the cohort of women in the retrieved studies will be evaluated case by case for possible inclusion.

No restriction on maternal age will be applied.

We will include only studies performed on women without or not carrying fetuses with congenital anomalies, including abnormal karyotype and/or genetic anomalies, and malformations. We will also include only studies performed on women without intrauterine infections and/or without history of drug or alcohol abuse. 
No other restriction on maternal clinical conditions will be applied.

\section{Interventions}

Based on current experimental treatments for FGR, ${ }^{1}$ we will consider the following therapeutic interventions:

- Heparin or low-molecular-weight heparin.

- Acetylsalicylic acid.

- Other antiplatelet agents (including ditazole, cloricromen, clopidogrel, ticlopidine, dipyridamole, carbasalate, epoprostenol, indobufen, iloprost, aloxiprin, eptifibatide, tirofiban, triflusal, beraprost, treprostinil, prasugrel, cilostazol, ticagrelor, cangrelor, vorapaxar and selexipag).

- Phosphodiesterase type 5 inhibitors.

- Phosphodiesterase type 3 inhibitors.

- Maternal VEGF gene therapy.

- Nanoparticles.

- MicroRNA.

- Statins.

- Nitric oxide donors.

- Hydrogen sulfide.

- Proton pump inhibitors.

- Melatonin.

- Creatine.

- N-acetylcysteine.

- Insulin-like growth factor 1 and 2 (IGF1 and IGF2).

Studies in which patients were co-treated with more than one above-mentioned treatment will be included, as well.

Additional pharmacological interventions, not listed above and detected by screening of retrieved references or in the bibliographies of evaluated studies, will be further considered; if pertinent, search strategies will be updated and related references will be retrieved.

There will be no restriction in the pregnancy week of beginning of the therapeutic intervention.

\section{Comparators}

We will consider studies comparing the effect of the above-mentioned interventions versus placebo or no treatment (considered together), or versus active control, that is, a second treatment listed above.

\section{Outcomes}

We will include only studies evaluating the primary efficacy outcome, that is, FGR. As the definition of FGR is based on biometric measures not always reported in details in published studies, we will accept the diagnosis of FGR provided by the authors of the studies.

In studies evaluating the above-mentioned primary outcome, also the following secondary efficacy outcomes will be considered (when reported):

- Preterm birth, defined as a delivery before completing 37 weeks of gestation.

- Fetal or neonatal death, including the events related to early or late pregnancy loss, and perinatal and early neonatal death. ${ }^{17}$
- Neonatal complications, including necrotising enterocolitis, respiratory distress syndrome, bronchopulmonary dysplasia, intraventricular haemorrhage, retinopathy of prematurity, periventricular leucomalakia and other conditions related to the abnormal growth and reported in the considered studies.

In studies reporting the primary outcome, also data related to placental lesions (ie, fetal and maternal vascular malperfusion) will be retrieved. ${ }^{18}$

As for safety outcomes, we will consider any side effect experienced by treated women or by fetuses or newborns in included studies; side effects will be defined based on authors' definitions.

In addition, in studies reporting the primary outcome, all maternal adverse events (such as pre-eclampsia, placental abruption, etc) will be evaluated.

Timing

There will be no timing restriction.

Setting

There will be no restriction by type of setting.

Language

We will include only articles written in the English language.

\section{Information sources and search strategy}

Electronic searches will be performed in the databases MEDLINE and Embase.

The MEDLINE search strategy is reported in online supplementary material 1 .

The MEDLINE search strategy will be adapted to the syntax and subject headings of the Embase.

Records will be retrieved on the same day from all sources.

The search strategy will be updated towards the end of the review, after being validated to ensure that the MEDLINE strategy retrieves a high proportion of eligible studies found through any means and indexed in MEDLINE.

\section{Study records}

Data management

Retrieved records will be managed using the software Endnote.

\section{Selection process}

Two review authors will independently screen the extracted records. The two review authors will independently identify studies for inclusion by screening titles and abstracts yielded by search, eliminating those deemed irrelevant. We will retrieve full-text articles for all references that at least one of the review authors will identify for potential inclusion. We will select studies for inclusion on the basis of review of full-text articles. We will resolve discrepancies through discussion.

Neither of the review authors will be blind to the journal titles or to the study authors or institutions. 


\section{Data collection}

Two review authors will independently extract data from the included studies.

Data abstracted will include demographic information, methodology, intervention details, all reported clinically relevant conditions and outcomes. Data will be extracted at the trial arm level, rather than the individual patient level. We will resolve discrepancies between authors through discussion.

\section{Data items}

Extracted data will include the name of the study authors and year of publication, the study design and characteristics (including single or double blinding and randomisation), the country in which participants were recruited, and eventual funding sources.

As for the population, we will extract the women's age, the specific risk factors for FGR and other clinically relevant comorbidities.

As for the intervention and the comparator, we will extract the active principle of the experimental intervention, its route of administration, the treatment dosage, the gestation week at time of treatment beginning and the duration of treatment.

We will extract the number of randomised participants, the number of participants included in the analysis, the number of participants with events for binary outcomes and the reported definition of outcomes, if appropriate. Whenever possible, we will use results from an intentionto-treat analysis.

\section{Outcomes and prioritisation}

The primary outcomes will be the number of women experiencing FGR out of the total number of treated patients.

The secondary outcomes will be the number of cases of preterm birth and of fetal or neonatal death, out of the total number of treated patients. For neonatal complications, we will consider the number of newborns with a given complication out of the total number of treated cases.

For efficacy outcomes, where Odds Ratio (ORs) or relative risks and related Confidence Intervals (CIs) are reported, these will be transformed to absolute numbers.

Placental characteristics will be considered out of the total number of cases for whom placental evaluation was available, focusing on villous branching abnormalities and maternal decidual arteriopathy.

Safety outcomes will be the number of patients (either mothers, fetuses or newborns) experiencing any adverse event out of the total number of treated patients. Adverse events will be identified based on specific authors' definitions, and will be classified using the Medical Dictionary for Regulatory Activities (MedDRA) classification, according to preferred terms and system organ class classification. ${ }^{19}$ Similarly, maternal adverse events (such as pre-eclampsia, placental abruption, etc) will be the number of women experiencing any event out of the total number of treated patients.

\section{Risk of bias}

Two review authors will independently assess the included studies for bias. To assess the risk of bias of included randomised controlled trials, we will follow the Cochrane Handbook for Systematic Reviews of Interventions. ${ }^{20}$ Specifically, we will assess the risk of bias in included trials for the following domains: selection (random sequence generation; allocation concealment); performance (blinding of participants and personnel); detection (blinding of outcome); attrition (incomplete outcome data); reporting (selective reporting); other unclear bias.

To assess the risk of bias of observational studies, we will follow the Newcastle-Ottawa Quality Assessment Scale. ${ }^{21}$ Specifically, for included cohort studies, we will consider the following domains: selection (representativeness of the exposed cohort; selection of the non-exposed cohort; ascertainment of exposure; absence of outcome of interest at start of study); comparability; outcome (assessment of outcome; appropriate length of follow-up; adequacy of follow-up of cohorts).

For each domain in the two tools, we will describe the procedures undertaken for each study, including verbatim quotes. A judgement as to the possible risk of bias on each domain will be made from the extracted information, rating from 'low risk' to 'high risk'.

The judgements will be made independently by two review authors; disagreement will be resolved first by discussion and then by consulting a third author.

We will compute graphic representations of potential bias within included studies, using the software RevMan V.5.3 (Review Manager 5.3).

\section{Data synthesis}

If studies are sufficiently homogeneous in terms of design and comparator, we will synthesise results using a NetMA, thus allowing to simultaneously combine evidence from both direct head-to-head comparisons and indirect evidence, that is, interventions compared through a common comparator. $^{22} 23$

\section{Measures of treatment effect}

All considered outcomes are based on dichotomous data. For all considered efficacy outcomes, we will perform a NetMA using a random-effects model within a frequentist framework. We will calculate pooled ORs combining the estimates reported in each study using random-effects Mantel-Haenszel method.

For safety outcomes, no quantitative synthesis will be performed, and the proportions of each reported adverse event will be described at study level. Similarly, all reported maternal adverse events will be described, and no quantitative analysis will be performed.

For placental lesions, data from included studies will be narratively synthetised according to the type of described lesions (lesions related to maternal vascular 
underperfusion versus fetal vascular underperfusion), and no quantitative analysis will be performed.

\section{Unit of analysis issues}

All analysis will be conducted per trial arm, rather than at individual patient level.

\section{Dealing with missing data}

When there are missing data, we will attempt to contact original study authors to obtain the relevant missing information. Important numerical data will be carefully evaluated. If missing data cannot be obtained, the study will be excluded from the related analysis.

\section{Assessment of heterogeneity}

We will evaluate the clinical heterogeneity by considering the variability in participants' features among studies and in study characteristics (study design, intervention, follow-up).

We will evaluate statistical heterogeneity across studies using the I-squared and Cochran's $Q$ tests, and publication bias using plots of SE against effect estimate (bias is likely to cause asymmetry in such plots), or using formal tests such as Egger one or similar.

If high levels of heterogeneity exist (I-squared $\geq 50 \%$ or $\mathrm{p}<0.1$ ), we will try to explain the source of heterogeneity by conducting subgroup or sensitivity analysis.

\section{Subgroup and sensitivity analyses}

Subgroup analysis will be conducted for timing of intervention (within vs after the first trimester of pregnancy). Additional subgroup analysis will be performed, if appropriate, according to the clinical characteristics of patients in included studies.

We will conduct a sensitivity analysis including only clinical trials versus only observational studies. If possible, a second sensitivity analysis will be performed including only high-quality clinical trials.

\section{Meta-biases}

To determine whether reporting bias is present in included clinical trials, we will evaluate whether the protocol of the clinical trial was published before recruitment of study patients. Specifically, for studies published after July 2005, we will screen the Clinical Trial Register at ClinicalTrials.gov. We will evaluate whether selective reporting of outcomes is present (outcome reporting bias). The potential for reporting bias will be evaluated using funnel plots (if $\geq 10$ studies are present).

\section{Confidence in cumulative estimate}

The quality of evidence will be judged using the Grading of Recommendations Assessment, Development and Evaluation working group scale, considering the domains of risk of bias, consistency, directness, precision and publication bias. Quality will be adjudicated as high, moderate, low or very low. ${ }^{20}$

\section{PATIENT AND PUBLIC INVOLVEMENT}

The project for this systematic review and NetMA was conceived within the frame of the CiaoLapo Onlus-a charity for support to stillbirth and perinatal loss in response to an unmet need suggested by healthcare professionals and patients.

\section{ETHICS AND DISSEMINATION}

There is no primary data collection involved in this study, thus research ethics approval is not required.

Results will be disseminated by release of findings in a peer-reviewed scientific journal, and by abstracts and speeches at international meetings and congresses.

Contributors $A B, N L$ and GC designed the study, drafted the PROSPERO protocol and the manuscript. LA, AM, CR and AV contributed to study design and provided critical revisions on the manuscript. As this is a protocol paper, the research has not yet been conducted, no data have been acquired or interpreted.

Funding The authors have not declared a specific grant for this research from any funding agency in the public, commercial or not-for-profit sectors.

Competing interests None declared.

Patient consent for publication Not required.

Provenance and peer review Not commissioned; externally peer reviewed.

Open access This is an open access article distributed in accordance with the Creative Commons Attribution Non Commercial (CC BY-NC 4.0) license, which permits others to distribute, remix, adapt, build upon this work non-commercially, and license their derivative works on different terms, provided the original work is properly cited, appropriate credit is given, any changes made indicated, and the use is non-commercial. See: http://creativecommons.org/licenses/by-nc/4.0/.

\section{REFERENCES}

1. Groom KM, David AL. The role of aspirin, heparin, and other interventions in the prevention and treatment of fetal growth restriction. Am J Obstet Gynecol 2018;218:S829-40.

2. Gordijn SJ, Beune IM, Thilaganathan B, et al. Consensus definition of fetal growth restriction: a Delphi procedure. Ultrasound Obstet Gynecol 2016;48:333-9.

3. McCowan LME, Thompson JMD, Cronin RS, et al. Going to sleep in the supine position is a modifiable risk factor for late pregnancy stillbirth; Findings from the New Zealand multicentre stillbirth casecontrol study. PLoS One 2017;12:e0179396.

4. Gardosi J, Kady SM, McGeown P, et al. Classification of stillbirth by relevant condition at death (ReCoDe): population based cohort study. BMJ 2005;331:1113-7.

5. Brosens JJ, Pijnenborg R, Brosens IA. The myometrial junctional zone spiral arteries in normal and abnormal pregnancies: a review of the literature. Am J Obstet Gynecol 2002;187:1416-23.

6. Levine RJ, Lam C, Qian C, et al. Soluble endoglin and other circulating antiangiogenic factors in preeclampsia. $N$ Engl J Med 2006;355:992-1005.

7. Lyall F, Robson SC, Bulmer JN. Spiral artery remodeling and trophoblast invasion in preeclampsia and fetal growth restriction: relationship to clinical outcome. Hypertension 2013;62:1046-54.

8. Sakamoto M, Osato K, Kubo M, et al. Early-onset fetal growth restriction treated with the long-acting phosphodiesterase-5 inhibitor tadalafil: a case report. J Med Case Rep 2016;10:317.

9. Lausman A, Kingdom J. Maternal Fetal Medicine Committee. Intrauterine growth restriction: screening, diagnosis, and management. J Obstet Gynaecol Can 2013;35:741-8.

10. "Institute of Obstetricians and Gynaecologists, Royal College of Physicians I, 'Directorate of Clinical Strategy and Health Service Executive Programmes'. Fetal growth restriction - recognition, diagnosis \& management. 2017.

11. 'Royal College of Obstetricians and Gynaecologists'. The Investigation and Management of the Small-for-Gestational-Age Fetus. 2014.

12. Vayssière $C$, Sentilhes $L$, Ego $A$, et al. Fetal growth restriction and intra-uterine growth restriction: guidelines for clinical practice from 
the French College of Gynaecologists and Obstetricians. Eur J Obstet Gynecol Reprod Biol 2015;193:10-18.

13. ACOG. ACOG Committee Opinion No. 743: Low-Dose Aspirin Use During Pregnancy. Obstet Gynecol 2018;132:e44-52.

14. Rodger MA, Gris JC, de Vries JIP, et al. Low-molecular-weight heparin and recurrent placenta-mediated pregnancy complications: a meta-analysis of individual patient data from randomised controlled trials. Lancet 2016;388:2629-41.

15. Shamseer L, Moher D, Clarke M, et al. Preferred reporting items for systematic review and meta-analysis protocols (PRISMA-P) 2015: elaboration and explanation. BMJ 2015;349:g7647.

16. Nardozza LM, Caetano AC, Zamarian AC, et al. Fetal growth restriction: current knowledge. Arch Gynecol Obstet 2017;295:1061-77.

17. Lawn JE, Blencowe H, Pattinson R, et al. Stillbirths: Where? When? Why? How to make the data count? Lancet 2011;377:1448-63.
18. Khong TY, Mooney EE, Ariel I, et al. Sampling and Definitions of Placental Lesions: Amsterdam Placental Workshop Group Consensus Statement. Arch Pathol Lab Med 2016;140:698-713.

19. MedDRA classification. https://www.meddra.org/how-to-use/basics/ hierarchy

20. Higgins JPT, Altman DG, Gotzsche PC, et al. The Cochrane Collaboration's tool for assessing risk of bias in randomised trials. BMJ 2011;343:d5928.

21. Wells GA, Shea B, O'Connell D, et al. The Newcastle-Ottawa Scale (NOS) for assessing the quality of nonrandomized studies in metaanalyses. Ottawa Hosp Res Inst 2013.

22. Caldwell DM, Ades AE, Higgins JP. Simultaneous comparison of multiple treatments: combining direct and indirect evidence. BMJ 2005;331:897-900.

23. Salanti G. Indirect and mixed-treatment comparison, network, or multiple-treatments meta-analysis: many names, many benefits, many concerns for the next generation evidence synthesis tool. Res Synth Methods 2012;3:80-97. 